\title{
A importância da escuta qualificada no cuidado clínico de enfermagem ao paciente oncológico
}

\section{The importance of qualified listening in clinical nursing care to onco- logical patients}

\author{
Beatriz Silva Passos' • Thais Martins Gomes de Oliveira ${ }^{2} \bullet$ Maria Luiza Rêgo Bezerra ${ }^{3}$ Andrey Hudson Intera- \\ minense Mendes de Araújo ${ }^{4}$
}

\begin{abstract}
RESUMO
Objetivo: descrever, de acordo com a literatura, o impacto da escuta qualificada realizada pelo enfermeiro no cuidado clínico com o paciente acometido pelo câncer. Metodologia: revisão integrativa, com busca de estudos produzidos e publicados entre 2015 e 2020, nas bases de dados Biblioteca Virtual em Saúde, Literatura Latino -Americana em Ciências da Saúde, Periódicos Capes e Scientific Eletronic Library Online, com os descritores controlados em português, em inglês e em espanhol. Também, foram acrescentados seis artigos a partir da busca manual no Google Achademics. A análise de conteúdo foi realizada a partir da identificação dos focos temáticos de onze artigos selecionados. Resultados: identificaram-se três categorias: enfermeiro como referência de apoio e conforto; capacitação e perfil profissional do enfermeiro que atua sobre as demandas clínicas oncológicas de seus pacientes; fatores capazes de dificultar e qualificar a escuta no cuidado clínico de enfermagem. Conclusão: a literatura evidenciou que a escuta é indispensável e deve ser adotada de forma qualificada na rotina de atuação do enfermeiro que atende pacientes oncológicos. Observou-se que a partir dessa adoção surge desse profissional um mediador redutor de incertezas e de angústias, deixando o cuidado clínico mais confortável e aceito. Palavras-Chave: Enfermagem Oncológica; Acolhimento; Enfermagem; Cuidados de Enfermagem; Relações Enfermeiro-Paciente.
\end{abstract}

\begin{abstract}
Objective: describes, according to the literature, an importance of qualified listening performed by nurses in clinical care for patients affected by cancer. Methodology: integrative review, looking for studies produced and properly published between 2015 and 2020, in the Virtual Health Library, Latin American Literature in Health Sciences, Capes Periodicals and Scientific Electronic Library Online, with controlled descriptors in Portuguese, English and Spanish.Also, six articles were added from the manual search on Google Achademics. The content analysis was performed based on the identification of the thematic focuses of eleven selected articles. Results: three categories were identified: nurse as a reference for support and comfort; training and professional profile of nurses who work on the clinical demands of their patients; factors capable of hindering and qualifying listening in clinical nursing care. Conclusion: the literature has shown that listening is indispensable and should be adopted in a qualified way in the routine of the nurses who work with cancer patients. It was observed that from this adoption, a mediator emerges from this professional to reduce uncertainty and anguish, making clinical care more comfortable and accepted.
\end{abstract}

Keywords: Oncology Nursing; User Embracement; Nursing; Nursing Care; Nurse-Patient Relations.

I Acadêmica no último semestre (8 período - Universidade Paulista do campus Brasília DF). E-mail: beatrizpassos09@gmail.com

2 Doutoranda e Mestre em Enfermagem- UnB. Especialista em Saude do Trabalho. Enfermeira - UnB. Professora do Curso de Enfermagem da Universidade Paulista - campus Brasília DF. E-mail: thaismmgomes@gmail.com

3 Doutoranda em Bioética e Mestrado em Enfermagem pela UnB. Docente do curso de Enfermagem da Universidade Paulista (UNIP), campus Brasília-DF. Email: marialuizaregobezerra@gmail.com

4 Mestre em Ciência e Tecnologias em Saúde - UnB. Especialista em Saúde da Família (UNESA). Enfermeiro - UnB. Professor do Curso de Enfermagem da Universidade Paulista - campus Brasília-DF. E-mail: profandreyh@gmail.com 


\section{INTRODUÇÃO}

O câncer é o principal problema de saúde pública em todo o mundo( ${ }^{(1)}$. É relevante o papel do enfermeiro nesse processo de atuação em escutar pacientes e familiares, principalmente pelo fato da equipe de enfermagem permanecer ao lado do paciente paliativo em tempo integral. Ainda pouco utilizada, a escuta tem se destacado como um importante recurso terapêutico em grande parte do processo saúde-doença. Conviver com o câncer gera um grande impacto na qualidade de vida dos pacientes acometidos. $O$ enfermeiro e sua equipe devem exercer essa abordagem de forma ativa, focando e valorizando o sujeito e as suas necessidades. A preocupação com o tempo dos atendimentos é um obstáculo para que a escuta seja feita da forma na qual deveria. Infelizmente, não operacionalizar corretamente a escuta é uma opção particular de muitos profissionais ou até mesmo por desconhecerem que a escuta terapêutica seja da sua área de atuação(2-4).

Em geral, a escuta terapêutica é bastante associada aos cuidado em saúde mental. A funcionalidade dela não é formada somente pelo ato de ouvir o sujeito, mas também de criar uma integração de confiança na comunicação e na relação profissional-paciente ${ }^{(4)}$.Também é formada pelo ato de desenvolver um plano de cuidado clínico, que possibilite a minimização de angústias pessoais, seja elas quais forem. Por essa razão, problematizar essa prática necessária altera negativamente a coleta dos dados necessárias, consequentemente, os resultados também. Felizmente, ao longo da construção do sistema de saúde no Brasil, estratégias e ações vieram para firmar a atenção e a gestão dos processos de produção de saúde. De tal forma que, a assistência ao cuidado oncológico e a valorização dos direitos dos usuários dos serviços fossem garantidos, na qual incluído na consulta de enfermagem está a escuta ativa e humanizada, valorizando o indivíduo como um todo ${ }^{(5)}$.

Dentre as competências de um enfermeiro, está nítido que efetuar a escuta ativa como uma boa prática de enfermagem, facilita $\circ$ trabalho e a rotina de toda a equipe. Avaliar o estado clínico com ênfase biopsicossocial de um paciente, coloca o enfermeiro como um agente receptor e também como um agente transportador que regista e assim dissemina as informações determinantes para os outros profissionais responsáveis. Os enfermeiros têm o papel na compreensão do cuidado da vida e no processo de viver do humano, garantindo uma assistência segura e, é dever da equipe de enfermagem prestar um cuidado e uma escuta de qualidade tratando o paciente com dignidade e com sigilo de suas informações pessoais ${ }^{(4-6)}$.
De acordo com os dados estatísticos do Ministério da Saúde relacionados ao câncer, divulgados em maio de 2020 pelo Instituto Nacional de Câncer (INCA), a incidência estimada, em homens, conforme a localização primária do tumor de próstata simboliza $29,2 \%$ comparado a todas as outras neoplasias, o que equivale a 65.840 casos novos no Brasil. Já em mulheres brasileiras, a localização primária do tumor de mamas simboliza uma incidência maior, e que comparado a todas as outras neoplasias, corresponde a $29,7 \%$, o que equivale a 66.280 casos novos $^{(7)}$. Em especial, nota-se que esses cânceres citados podem afetar diretamente o estado físico e psicológico dos pacientes. Por esse motivo, a escuta terapêutica é muito importante pois inclui o sujeito no contexto do cuidado, dando a ele seu direito de estar envolvido em decisões acerca de seu tratamento e tão associado à sua autoestima ${ }^{(8)}$.

A escolha do tema foi baseada na intenção de demonstrar que, mesmo sendo pouco aplicada, a escuta qualificada, além de não gerar custos financeiros, gera resultados quando utilizada como estratégia na assistência. A escuta no ambiente oncológico é uma ferramenta necessária pois cria um vínculo entre os pacientes, as famílias, os profissionais e as unidades. Os enfermeiros que adotam esta prática em sua rotina e que buscam aumentar a autoestima de seus clientes conseguem planejar melhor as ações e aprimorar a qualidade da interação profissional/paciente/família/ instituição, obtendo maior aceitação do paciente ao tratamento quimioterápico ${ }^{(8)}$.

O presente estudo torna-se relevante em razão de que na avaliação clínica oncológica, o enfermeiro deve estar atento aos sinais e sintomas dos seus pacientes, coletando os dados importantes e aberto a enxergar uma realidade que nem sempre é igual a dele. Tratar o acompanhante como um forte comunicador de informações também faz parte do atendimento de enfermagem e aumenta as chances de entender o processo saúde-doença em um contexto clínico. Dessa forma, motivar a escuta significa absorver e entender quais são as dificuldades da rotina de tratamento e que afetam as necessidades básicas desses indivíduos ${ }^{(9)}$.

O objetivo deste estudo é descrever, de acordo com a literatura, o impacto da escuta qualificada realizada pelo enfermeiro no cuidado clínico com o paciente acometido pelo câncer. Elencar a finalidade e a importância dessa escuta na rotina dos serviços de enfermagem e de que modo é desenvolvida, compreendendo o papel do enfermeiro no contexto oncológico, reforçando sempre a ideia de que a capacidade para a escuta faz parte da filosofia de enfermagem. Valorizar o que o paciente carrega em si: medos, crenças e princípios que devem ser 
respeitados e levados em consideração pelo enfermeiro que irá colher os dados e analisá-los. Incluir as condições, o ambiente e o contexto em que esse paciente vive, pois tais fatores devem fazer parte do cuidado clínico que será planejado a ele. Tendo como questão norteadora: "De que forma a escuta qualificada realizada pelo enfermeiro pode impactar no cuidado clínico de pacientes acometidos pelo câncer?"(9).

\section{MÉTODO}

Revisão integrativa sobre a estratégia de atuação profissional e comprometida do enfermeiro por meio da escuta qualificada. Essa revisão foi desenvolvida em sete etapas, descritas a seguir: escolha do tema e desenvolvimento da pergunta norteadora; amostragem (definição dos critérios de inclusão e exclusão); pesquisa dos estudos (estratégia PICO); categorização dos estudos (classificação dos dados a serem extraídos dos estudos selecionados); avaliação dos estudos (análise crítica dos estudos escolhidos); exposição dos resultados (discussão dos resultados essenciais) e apresentação da revisão/ síntese do conhecimento acerca do tema investigado ${ }^{(10)}$.

$\mathrm{Na}$ etapa de pesquisa, a população do estudo são pacientes oncológicos, com interesse e indicação da postura profissional do enfermeiro durante sua atuação baseada no ouvir e na interpretação dos sinais apresentados por seus clientes, não-intervenção de comparação, com resultado na construção de uma conduta de escuta. $\mathrm{Na}$ etapa de categorização dos artigos escolhidos, foram utilizados os seguintes itens: autor, ano de publicação, título, delineamento, local de realização do estudo, idioma de publicação, nível de evidência (conforme padronizado pelo Oxford Centre for Evidence-based Medicine, que foi o método utilizado para avaliar a qualidade da evidência), objetivos, resultados e conclusões do estudo. $O$ método de Oxford classifica a evidência em níveis: Ia, Ib, Ic, 2a, 2b, 2c, 3a, 3b, 4 e 5, considerando o ensaio clínico randomizado como delineamento forte e o estudo de coorte como delineamento relativamente mais fraco, mas que com investigações bem feitas alcançam resultados de alta credibilidade. $\mathrm{Na}$ avaliação, na interpretação e na síntese dos estudos foi utilizado a análise categorial temática a partir da identificação dos núcleos de sentido(II).

A busca dos artigos foi realizada nas coleções eletrônicas Biblioteca Virtual em Saúde, Literatura Latino-Americana em Ciências da Saúde, Periódicos Capes, Scientific Eletronic Library Online e Literatura Cinzenta no Google Achademic. Na busca dos artigos foram utilizados os seguintes descritores: Oncology Nursing OR Enfermagem Oncológica AND User Embracement OR Acolhimento AND Nursing OR Enfermagem AND Nursing Care OR Cuidados de Enfermagem AND Nurse-Patient Relations OR Relações Enfermeiro-Paciente, que estão contidos nos Descritores em Ciências da Saúde, o qual permite o uso de terminologias comuns para a pesquisa, proporcionando, a recuperação das informações necessárias para o desenvolvimento da revisão de forma consistente ${ }^{(12)}$.

Os critérios utilizados para a seleção e a inclusão dos artigos foram: artigos indexados nas bases de dados descritas anteriormente; artigos publicados em português, inglês e espanhol; entre os anos de 2015 e 2020, que abrangessem a temática do estudo e que pudessem enriquecer de definições e informações o estudo; artigos originais e em texto completo disponível que respondiam à pergunta norteadora. A verificação desse último critério de inclusão foi realizada após a leitura dos títulos e dos resumos e após a leitura integral dos artigos selecionados. De forma necessária, também foram incluídos artigos a partir de busca manual no Google Achademic, pois esses não faziam parte dos resultados da busca automática, mas atendiam aos critérios de inclusão e traziam reflexões e contribuições relevantes acerca da temática trabalhada. Dentre os critérios de exclusão, estão os artigos que não respondem a pergunta norteadora, que não abordassem a escuta qualificada de enfermagem e/ou que estão indexados em mais de uma base ou duplicados na mesma base.

O uso da revisão integrativa no contexto da escuta qualificada de enfermagem proporciona uma análise, sobre aspectos não tecnológicos e não farmacológicas que o enfermeiro deve dominar, como: a boa comunicação e a recepção atenta das informações durante toda sua rotina de trabalho, na atualidade do cuidado com seus pacientes acometidos pelo câncer.

\section{RESULTADOS}

Os resultados estão apresentados de forma descritiva e por meio de fluxogramas, tabelas e quadros com o objetivo de captar as evidências sobre a importância de uma escuta qualificada. A Figura I traz o fluxograma de busca da pesquisa, segundo PRISMA ${ }^{(13)}$. A categorização desses resultados está apresentada no Quadro I.A síntese de cada obra da temática abordada está descrita no Quadro 2, com os principais resultados/conclusão do estudo relacionados a pesquisa.

$O$ cheque liste do PRISMA foi seguido. Conforme etapas descritas na Figura I, após a busca nas bases de dados, foram encontrados 124 artigos. Dos quais foram selecionados 57 artigos, conforme descritos na triagem da seleção. Observados os critérios de elegibilidade, 16 artigos foram excluídos pelo fator de duplicidade. Diante disso, considerando os estudos que apresentam características da temática estudada e que adequadamente respondem a questão norteadora, foram incluídos II artigos na análise qualitativa. 
Estudos identificados pela pesquisa nas bases de dados.

BVS ( $N=18)$, LILACS ( $N=59$ ), Periódicos Capes $(N=36)$, SCIELO ( $N=5)$, Google Achademics ( $N=6)$.

$(\mathrm{N}=\mathbf{1 2 4})$

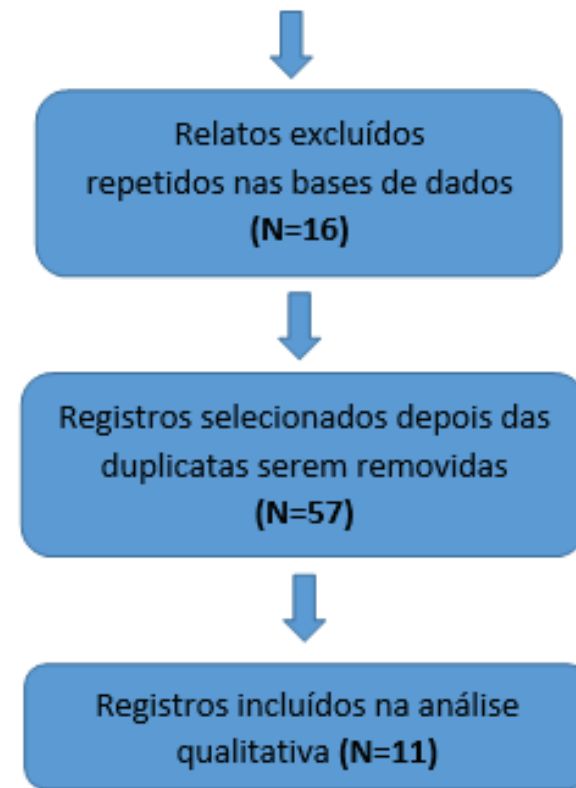

Registros excluídos por não atenderem o tema proposto,

por não responderem a pergunta norteadora e/ou por não acrescentarem aspectos acerca da temática de escuta qualificada de enfermagem

$(\mathrm{N}=\mathbf{4 6})$

FIGURA 1 - Fluxograma do processo de identificação, seleção e inclusão dos estudos elaborados a partir da recomendação PRISMA(13).

Fonte: Elaborado pelos autores.

TABELA 1 - Número de artigos obtidos nas bases de dados nos anos 2015 a 2020

\begin{tabular}{l|c|c|c|c|c}
\hline $\begin{array}{c}\text { Base } \\
\text { de Dados }\end{array}$ & Artigos Encontrados & $\begin{array}{c}\text { Artigos } \\
\text { Repetidos }\end{array}$ & Artigos Selecionados & $\begin{array}{c}\text { Artigos } \\
\text { Excluídos }\end{array}$ & $\begin{array}{c}\text { Artigos } \\
\text { Incluídos }\end{array}$ \\
\hline BVS & 18 & 2 & 10 & 9 & 1 \\
\hline LILACS & 59 & 9 & 25 & 25 & 0 \\
\hline PERIÓDICOS CAPES & 36 & 4 & 12 & 2 & 2 \\
\hline SCIELO & 5 & 1 & 6 & 0 & 6 \\
\hline GOOGLE ACHADEMIC & 6 & 0 & 57 & 46 & 11 \\
\hline Total & 124 & 16 & 4 & & 2 \\
\hline
\end{tabular}

Nota: Descrição das buscas dos artigos nas bases de dados.

Fonte: Elaborado pelos autores.

QUADRO 1 - Descrição dos estudos incluídos da revisão integrativa, segundo autores, ano de publicação, título, delineamento, local, idioma e nível de Evidência Científica por Tipo de Estudo(14) - "Oxford Centre for Evidence-based Medicine".

\begin{tabular}{|c|l|c|c|c|c|}
\hline Autores/Ano & Título & Delineamento & Local//dioma & $\begin{array}{c}\text { Nível de } \\
\text { Evidência }\end{array}$ & Vínculo terapêutico \\
\hline Ribeiro et al, 2016 (2). & $\begin{array}{l}\text { Assistência de } \\
\text { enfermagem ao paciente } \\
\text { oncológico hospitalizado: } \\
\text { diagnósticos e } \\
\text { intervenções } \\
\text { relacionadas ás } \\
\text { necessidades } \\
\text { psicossociais e } \\
\text { espirituais. }\end{array}$ & $\begin{array}{c}\text { Quali-quantitativo, } \\
\text { exploratório-descritivo }\end{array}$ & $\begin{array}{c}\text { Brasil/ } \\
\text { Português }\end{array}$ & $\begin{array}{l}\text { Busca pelo equilíbrio } \\
\text { do corpo e da alma e } \\
\text { a continuidade da vida } \\
\text { com o maior grau de } \\
\text { qualidade possível }\end{array}$ \\
\hline
\end{tabular}




\begin{tabular}{|c|c|c|c|c|c|}
\hline $\begin{array}{l}\text { Markus et al, } \\
2017^{(4)}\end{array}$ & $\begin{array}{l}\text { A atuação do enfermeiro } \\
\text { na assistência ao } \\
\text { paciente em cuidados } \\
\text { paliativos. }\end{array}$ & Qualitativo, descritivo & $\begin{array}{c}\text { Brasil/ } \\
\text { Português }\end{array}$ & 5 & $\begin{array}{l}\text { Alívio da tensão } \\
\text { inerente à gravidade } \\
\text { da situação, } \\
\text { protegendo a } \\
\text { dignidade e os } \\
\text { valores do paciente } \\
\text { paliativado(4). }\end{array}$ \\
\hline $\begin{array}{c}\text { Menezes et al, } \\
2020^{(5)} \text {. }\end{array}$ & $\begin{array}{l}\text { A relação enfermeiro- } \\
\text { paciente oncológico } \\
\text { em uma abordagem } \\
\text { holística. }\end{array}$ & Qualitativo & Brasil/ Português & 5 & $\begin{array}{l}\text { Sentimentos dos } \\
\text { familiares e clientes } \\
\text { oncológicos permitidos } \\
\text { à fala, identificando e } \\
\text { ajudando-os na busca } \\
\text { de soluções, com } \\
\text { atitudes de aceitação } \\
\text { e escuta(5). }^{(5)}\end{array}$ \\
\hline Óscar et al, 2015(15). & $\begin{array}{l}\text { Atención al detalle, un } \\
\text { requisito para el cuidado } \\
\text { humanizado. }\end{array}$ & $\begin{array}{c}\text { Qualitativo } \\
\text { fenomenológico } \\
\text { interpretativo }\end{array}$ & $\begin{array}{l}\text { Medellín } \\
\text { (Colômbia)/ } \\
\text { Espanhol }\end{array}$ & $3 A$ & $\begin{array}{l}\text { Cuidado, apoio e ajuda } \\
\text { com comprometimento } \\
\text { e satisfação em fazê-lo } \\
\text { e não apenas com a } \\
\text { intenção de cumprir } \\
\text { um trabalho, horário } \\
\text { ou atribuição(15). }\end{array}$ \\
\hline $\begin{array}{l}\text { Garcia et al, } \\
2018^{(16)}\end{array}$ & $\begin{array}{l}\text { The effect of therapeutic } \\
\text { listening on anxiety and } \\
\text { fear among surgical } \\
\text { patients: randomized } \\
\text { controlled trial. }\end{array}$ & $\begin{array}{l}\text { Ensaio clínico } \\
\text { randomizado } \\
\text { (aleatorizado e } \\
\text { controlado) }\end{array}$ & Inglês & $2 B$ & $\begin{array}{l}\text { Diminuir a ansiedade } \\
\text { e o medo cirúrgico, } \\
\text { presentes em } \\
\text { pacientes que } \\
\text { aguardam um } \\
\text { procedimento } \\
\text { cirúrgico }^{(16)} \text {. }\end{array}$ \\
\hline $\begin{array}{l}\text { Anacleto et al, } \\
2020^{(18)}\end{array}$ & $\begin{array}{l}\text { Cuidado de enfermagem } \\
\text { humanizado ao paciente } \\
\text { oncológico: revisão } \\
\text { integrativa. }\end{array}$ & Qualitativo & Brasil/ Português & 5 & $\begin{array}{l}\text { Compreender as } \\
\text { vivências, promover } \\
\text { conforto e alivio, } \\
\text { incluída sua condição } \\
\text { básica de ser humano } \\
\text { de acordo com as suas } \\
\text { potencialidades }{ }^{(18)} \text {. }\end{array}$ \\
\hline $\begin{array}{l}\text { Catapreta et al, } \\
2020^{(20)}\end{array}$ & $\begin{array}{l}\text { A comunicação na } \\
\text { unidade de terapia } \\
\text { intensiva oncológica: } \\
\text { Uma revisão } \\
\text { sistemática sobre os } \\
\text { vieses que interferem } \\
\text { e ou participam na } \\
\text { comunicação entre } \\
\text { enfermeiros e pacientes } \\
\text { oncológicos. }\end{array}$ & Qualitativo, descritivo & Brasil/ Português & 5 & $\begin{array}{l}\text { Valorização do } \\
\text { compreender e } \\
\text { ser compreendido } \\
\text { com observação do } \\
\text { gestual, expressões } \\
\text { faciais e reflexos } \\
\text { involuntários } \\
\text { (20). }\end{array}$ \\
\hline $\begin{array}{c}\text { Nascimento, } \\
2019^{(21)}\end{array}$ & $\begin{array}{l}\text { Influências sociais } \\
\text { e emocionais da } \\
\text { humanização a } \\
\text { assistência ao paciente } \\
\text { oncológico. }\end{array}$ & Investigação qualitativa & $\begin{array}{c}\text { Brasil/ } \\
\text { Português }\end{array}$ & 5 & $\begin{array}{l}\text { Cuidados que } \\
\text { privilegiem, dentre } \\
\text { outros, aspectos } \\
\text { psicológicos. Atitude } \\
\text { de aceitação e de } \\
\text { escuta associada } \\
\text { à criação e à } \\
\text { manutenção de } \\
\text { um ambiente } \\
\text { terapêutico } \\
\text { (21). }\end{array}$ \\
\hline Lira et al, $2019^{(24)}$. & $\begin{array}{l}\text { Assistência do } \\
\text { enfermeiro no } \\
\text { atendimento domiciliar } \\
\text { em pacientes } \\
\text { oncológicos. }\end{array}$ & $\begin{array}{c}\text { Qualitativo, exploratório } \\
\text { e descritivo }\end{array}$ & $\begin{array}{l}\text { Brasil/ } \\
\text { Português }\end{array}$ & 5 & $\begin{array}{l}\text { Criação de vínculo } \\
\text { através de orientação } \\
\text { para o desenvolver } \\
\text { de um bom } \\
\text { atendimento(24). }\end{array}$ \\
\hline
\end{tabular}




\begin{tabular}{|c|c|c|c|c|c|}
\hline $\begin{array}{l}\text { Brandão et al, } \\
2020^{(26)}\end{array}$ & $\begin{array}{l}\text { Assistência de } \\
\text { enfermagem para } \\
\text { pacientes oncológicos } \\
\text { em cuidados paliativos: } \\
\text { importância da interação } \\
\text { familiar no tratamento. }\end{array}$ & Qualitativo & $\begin{array}{c}\text { Brasil/ } \\
\text { Português }\end{array}$ & 5 & $\begin{array}{l}\text { Escutar mais do que } \\
\text { falar, para expressão } \\
\text { de sentimentos } \\
\text { associados } \\
\text { ao paciente. } \\
\text { Aperfeiçoamento } \\
\text { de habilidades } \\
\text { técnico-científicas } \\
\text { e capacidade de } \\
\text { percepção das } \\
\text { necessidades } \\
\text { de forma a oferecer } \\
\text { cuidados de en- } \\
\text { fermagem com } \\
\text { qualidade } \\
\text { (26). }\end{array}$ \\
\hline $\begin{array}{l}\text { Picollo et al, } \\
2019^{(29)} \text {. }\end{array}$ & $\begin{array}{l}\text { A atenção do enfermeiro } \\
\text { ao paciente em cuidado } \\
\text { paliativo. }\end{array}$ & Qualitativo & $\begin{array}{l}\text { Brasil/ } \\
\text { Português }\end{array}$ & 5 & $\begin{array}{l}\text { Oferta de qualidade } \\
\text { de vida e atenção } \\
\text { às demandas do } \\
\text { paciente, ofertando } \\
\text { espaço para acolher o } \\
\text { escutado }{ }^{(29)} \text {. }\end{array}$ \\
\hline
\end{tabular}

QUADRO 2 - Síntese temática abordada nas obras.

\begin{tabular}{|c|c|}
\hline Objetivo & Principais Resultados/Conclusão \\
\hline $\begin{array}{l}\text { Identificar os diagnósticos e intervenções de } \\
\text { enfermagem acerca } \\
\text { das necessidades psicossociais e psicoespirituais de } \\
\text { pacientes oncológicos }{ }^{(2)} \text {. }\end{array}$ & $\begin{array}{l}\text { Os diagnósticos } \\
\text { identificados concentraram-se nos domínios de Autopercepção, Papéis } \\
\text { e relacionamento, Enfrentamento total ao estresse, Conforto e Princípios } \\
\text { da vida. Evidenciaram-se as intervenções de enfermagem: melhora da } \\
\text { autopercepção, promoção de esperança, melhora do enfrentamento, } \\
\text { presença, fortalecimento da autoestima, manutenção do processo familiar, } \\
\text { melhora da socialização, aumento da segurança, apoio emocional, } \\
\text { escutar ativamente, apoio espiritual, melhoria da imagem corporal e } \\
\text { administração de analgésico(2). }\end{array}$ \\
\hline $\begin{array}{l}\text { Investigar a atuação do enfermeiro na assistência ao } \\
\text { paciente em Cuidados Paliativos }{ }^{(4)} \text {. }\end{array}$ & $\begin{array}{l}\text { A atuação do enfermeiro na assistência ao paciente em cuidados paliativos é } \\
\text { extremamente relevante, principalmente pela equipe de enfermagem que permanece } \\
\text { ao lado do paciente paliativa do em tempo integral. O profissional ainda realiza a } \\
\text { interface entre equipe de saúde e familiares, com respeito à condição humana e à } \\
\text { qualidade de vida desses pacientes }{ }^{(4)} \text {. }\end{array}$ \\
\hline $\begin{array}{l}\text { Apresentar a importância de demonstrar a } \\
\text { relação entre o enfermeiro e o paciente no apoio, } \\
\text { fortalecimento e encorajamento em todas as fases } \\
\text { da doença, mostrando-lhes a importância de adesão } \\
\text { ao tratamento }{ }^{(5)} \text {. }\end{array}$ & $\begin{array}{l}\text { A atuação dos enfermeiros não está voltada simplesmente à doença física do } \\
\text { paciente. Ao contrário, tem um olhar amplo que envolve interação assistencial e } \\
\text { humanística ao doente e familiar durante o tratamento oncológico. Assim, o vínculo } \\
\text { de confiança entre enfermeiro-paciente surge da utilização de estratégias, por vezes } \\
\text { inovadoras, deste profissional para minimizar situações de sofrimento e incertezas } \\
\text { em relação aos procedimentos adotados }{ }^{(5)} \text {. }\end{array}$ \\
\hline $\begin{array}{l}\text { Compreender o significado da experiência do } \\
\text { cuidado humanizado para os participantes }{ }^{(15)} \text {. }\end{array}$ & $\begin{array}{l}\text { O acolhimento sem discriminação, o interesse em cuidar, ouvir as reclamações, o } \\
\text { cuidar em todas as esferas e o envolvimento da família são detalhes essenciais no } \\
\text { cuidado humanizado }{ }^{(15)} \text {. }\end{array}$ \\
\hline $\begin{array}{l}\text { Investigar o efeito da escuta terapêutica sobre a } \\
\text { ansiedade estado e os medos relacionados à cirurgia } \\
\text { em pacientes no pré-operatório de cirurgia de câncer } \\
\text { colorretal }^{\left({ }^{(16)} \text {. }\right.}\end{array}$ & $\begin{array}{l}\text { Na comparação das variáveis nos grupos controle e intervenção no momento nos } \\
\text { momentos pré e pós-intervenção, constataram-se diferenças entre os momentos no } \\
\text { grupo controle para as variáveis cortisol, frequência de pulso e medos relacionados } \\
\text { a cirurgia, com redução dos valores dessas variáveis }{ }^{(16)} \text {. }\end{array}$ \\
\hline $\begin{array}{l}\text { Levantar e discutir vieses que possam interferir ou } \\
\text { participar na comunicação entre os profissionais de } \\
\text { enfermagem e os pacientes oncológicos internados } \\
\text { na unidade de terapia intensiva }{ }^{(20)} \text {. }\end{array}$ & $\begin{array}{l}\text { A habilidade de comunicação pode permitir novas reflexões e discussões } \\
\text { proporcionando melhorias na assistência. As ferramentas de auxílio podem fornecer } \\
\text { eficácia no atendimento, porém, a busca por aperfeiçoamento neste processo é } \\
\text { fundamental à melhor assistência, refletindo no tratamento e na reabilitação do } \\
\text { paciente oncológico internado }{ }^{(20)} \text {. }\end{array}$ \\
\hline
\end{tabular}


O objetivo desse artigo direciona-se aos aspectos sociais e emocionais de humanizar o pensar e o agir do profissional da saúde, especificamente, a enfermagem no intuito de compreender o significado da terapêutica quimioterápica, isto, com bases e estratégias de integração e inter-relação humana das equipes ${ }^{(21)}$.
O processo do cuidar durante todo o tratamento oncológico, envolve primordialmente a equipe de enfermagem, pois são eles os responsáveis pela maior parte do tempo de prestação desse cuidado, envolvendo uma relação de vínculo, empatia, escuta qualificada, e todos os outros fatores de aspectos emocionais, não se limitando somente ao tratamento quimioterápico, o que é muito importante para a qualidade da assistência, no controle social e emocional à humanização(21).

\section{Informar a importância da assistência de} enfermagem no atendimento domiciliar, informando o acompanhante fixo e familiares formas de comportamentos e métodos para identificar possíveis alterações no quadro de pacientes oncológico ${ }^{(24)}$.

Mostrar a importância da interação familiar e da assistência de enfermagem a fim de tratar da saúde física e mental do paciente oncológico ${ }^{(26)}$.
O enfermeiro tem um papel fundamental na articulação entre família e equipe multiprofissional, cuidando do paciente para que este alcance sua autonomia ou morra com dignidade ${ }^{(24)}$.

Conclui-se que através das produções científicas encontradas nesse estudo o objetivo do cuidado paliativo não é curar o paciente e sim promover conforto e alívio das sintomatologias do câncer durante o processo da morte. Por fim, neste processo do cuidar, evidencia-se que integrar a família no tratamento ao paciente oncológico paliativo é uma estratégia para promoção de conforto do enfermo e de seus familiares ${ }^{(26)}$.

Conhecer a produção científica em relação a enfermagem acerca dos cuidados paliativos, identificar o papel do enfermeiro frente aos cuidados paliativos, elencar as principais competências do profissional para torna-se apto a atuação em cuidados paliativos e verificar a importância da equipe multidisciplinar em cuidados paliativos ${ }^{(29)}$.

Observou-se a importância do trabalho do enfermeiro na equipe multidisciplinar atendendo as demandas do paciente de forma integral(29).
Através da análise dos estudos e da coleta de informações, emergiram-se três principais categorias temáticas: a) enfermeiro como referência de apoio e conforto; b) capacitação e perfil profissional do enfermeiro que atua sobre as demandas clínicas oncológicas de seus pacientes; c) fatores capazes de dificultar e qualificar a escuta no cuidado de enfermagem.

No que concerne ao método eleito pelos autores para a investigação, dos onze artigos de pesquisa analisados na presente revisão, aponta-se que três tratam-se de estudos transversais, o que simboliza $27 \%$ de toda a pesquisa. A população geral de um desses estudos está descrita da seguinte forma: levantamento de dados em prontuários de pacientes oncológicos internados em unidade hospital. O outro estudo possui população selecionada por amostragem proposital. Dessa população, 6 eram profissionais da saúde que tiveram experiências como pacientes, 7 eram parentes próximos de pacientes hospitalizados e outros 3 eram enfermeiras. Esses 16 participantes foram submetidos às entrevistas conversacionais em profundidade, com duração de 60 a 90 minutos, possibilitando compartilhar suas experiências acerca dos detalhes que são importantes para a relação enfermeiro-paciente ${ }^{(15)}$. A população geral do terceiro estudo transversal trata-se da seguinte forma: 50 pacientes hospitalizados para tratamento cirúrgico de câncer colorretal, maiores de 18 anos de idade, submetidos a interação durante 30 minutos com a pesquisadora sobre preocupações, medos, dúvidas ou sobre qualquer outro assunto que $o$ paciente quisesse $\operatorname{tratar}^{(16)}$.

\section{DISCUSSÃO}

$\mathrm{Na}$ busca para compreender o significada da palavra "escuta", verifica-se que ela deriva do verbo "escutar, do latim auscultare, que quer dizer tornar-se ou estar atento para ouvir; dar ouvidos a aplicar o ouvido com atenção para perceber ou ouvir e ouvir"(17). A dinâmica do esforço para entender corretamente o que o outro diz, faz parte da rotina diária dos profissionais de saúde, em especial, da rotina dos enfermeiros e de sua equipe com os pacientes oncológicos. Ouvir com interesse e respeito é uma atitude terapêutica. Quem está sendo ouvido sente-se aliviado quando compreendido e ajudado.

A escuta é parte do processo que se realiza nas interações mais humanas da profissão. Experiências pessoais são partilhadas e por isso deve ser tratada como uma estratégia valorosa na relação profissional-cliente. Além disso, a escuta deve ser desempenhada de forma integral e humanizada, o olhar atento do enfermeiro deve enxergar seu cliente como um todo, abrangendo a empatia no momento de ouvir e de se colocar no lugar e nas condições vividas por seus pacientes em tratamento cancerológico. A escuta eficiente e qualificada é a assistência adequada da linguagem verbal e não-verbal durante todo esse tratamento, e em todo local de assistência, seja ele hospitalar ou não.A forma como os profissionais de saú- 
de agem em todo esse processo, interfere em como os pacientes reagem ao tratamento e ao autocuidado de suas enfermidades ${ }^{(18)}$.

A forma como a linguagem é utilizada pode afetar negativamente ou positivamente o diálogo profissionalcliente.A linguagem não verbal é tão importante quanto a linguagem verbal. Pacientes acometidos pelo câncer vivenciam um conjunto de incertezas, por essa razão, os gestos designados pelo enfermeiro durante a entrevista podem interferir nas respostas ${ }^{(18)}$. Para que haja uma boa abordagem, o profissional deve estar disposto a entender os sentidos e dimensões do câncer e a respeitar as ideologias, experiências, crenças e situações sentidas por cada paciente. Essa enfermidade específica, os levaram ao serviço de saúde, e ao receber estes pacientes, cada detalhe deve ser levado em consideração, para que assim, seja aplicado um plano de cuidados individual e coerente com os problemas existentes. O desejável é que os problemas reais fossem os mesmos encontrados e interpretados pelo enfermeiro, pois assim, poderão ser solucionados e resolvidos da maneira mais adequada. Por esta razão é necessário pontuar a aplicabilidade e importância da boa escuta para o processo de recuperação da doença ${ }^{(19)}$.

\section{- Enfermeiro como referência de apoio e con- forto:}

Quando a confiança é estabelecida, paciente e família encontram conforto para falarem sobre experiências e impressões mais pessoais da doença. Cabe ao enfermeiro agir sobre suas necessidades de forma respeitosa, compreender de forma holística, receber cada queixa, valorizando a opinião e a forma de pensar de seu paciente ${ }^{(19)}$.A humanização da enfermagem existe para mostrar que o paciente não é apenas um acometido pelo câncer ou um dado. Cada paciente deve ser visto de forma individualizada e enxergado como um todo. Isso tudo são fatores que aumentam a eficiência da escuta em saúde, principalmente quando incluímos uma boa interação enfermeiro-paciente na humanização do cuidado(20).

O paciente com câncer passa por vários desafios e medos desencadeados pela mudança de vida que a doença causou. Cada paciente possui a sua peculiaridade marcada por fatores físicos e psicológicos. Dessa forma, como a equipe de enfermagem está a frente da assistência e passa a maior parte do tempo com o paciente, $\circ$ enfermeiro é visto como um intermediador do alívio das angústias. Assim, o paciente cria uma expectativa de que é esse o profissional que poderá ouvir e entender o que se passa ${ }^{(20)}$.

Pela necessidade do diálogo nas atividades de saúde, surge a comunicação que para ser efetiva, exige a escuta. Para haver um impacto positivo na assistência prestada, o enfermeiro precisa saber ouvir com flexibilidade, identificando e compreendendo o que o seu cliente diz e, às vezes, interpretando o que ele quer te dizer. Existem algumas razões que tornam a escuta terapêutica relevante na prática em saúde, e uma delas é: o sofrimento que é produzido pela angústia de não entender o que se passa em seu próprio corpo e a dificuldade de expor isso à alguém (seja por vergonha ou medo do julgamento alheio). Esse sofrimento podem gerar graves consequências psíquicas, principalmente quando associados ao stress $^{(21)}$.

Para que o paciente tenha segurança em relação ao tratamento é necessário que sinta-se confortável. Por isso é tão importante para o enfermeiro transparecer confiança e estar conectado aos sentimentos e preocupações do paciente, pois assim, ele sentirá que está situado e incluído nas decisões sobre sua saúde fisiológica e mental ${ }^{(21)}$. Isso é extremamente importante para a satisfação do cliente e para a interação desenvolvida durante o cuidado. Ou seja, contribuirá de forma favorável na resolução dos problemas e das alterações causadas pelo câncer, auxiliando a prática de enfermagem juntamente com o trabalho da equipe multidisciplinar. $O$ modo de aceitação terapêutica depende diretamente se a busca por apoio foi bem correspondida ${ }^{(22)}$.

\section{- Capacitação e perfil profissional do enfermei- ro que atua sobre as demandas clínicas onco- lógicas de seus pacientes}

Atuar com profissionalismo e comprometimento por meio da escuta é estabelecer um vínculo primordial e indispensável, pois quanto mais confiança há, melhor será a troca de informações e o enfermeiro estará cada vez mais conectado ao paciente acometido e ao câncer a ser tratado. $\mathrm{Na}$ busca pela qualificação das relações, nasce a inclusão da escuta como parte do acolhimento. Acolhimento no qual é usado como ferramenta aplicável no ambiente hospitalar ou no atendimento domiciliar (Home Care). É notável que grande parte dos atendimentos que possuem resultados negativos foram por falhas de um enfermeiro que faltou com a boa escuta e conduta ética. Resultados que geralmente os profissionais costumam associar a falta de recursos ${ }^{(21-23)}$.

No ambiente de cuidado oncológico, ser empático é uma virtude na qual o enfermeiro deve ter, pois assim ele se coloca em uma realidade que não vivencia, e estará sempre tentando compreender o que o outro expressa. Alcançar um comunicação satisfatória é eficaz para contribuir no acompanhamento dos pacientes ${ }^{(24)}$. $O$ desejo do enfermeiro em envolver-se e interagir de forma respeitosa, faz com que a orientação seja recebida de forma muito mais aceitável. $O$ processo de diagnóstico e recuperação da saúde devem ser repassados pelo enfermeiro de forma clara e formal, e para isso é preciso que os 
profissionais que irão desempenhar esse papel, estejam cientes da importância da escuta qualificada. Em relação a assistência, utilizar das técnicas de relação interpessoal é produtivo para aumentar a dinâmica e confiança da relação profissional-cliente ${ }^{(25)}$.

Atender os usuários dos serviços de saúde com uma boa escuta e realizar o registro legal das informações que foram coletadas e selecionadas, são ações facilitadoras a comunicação e diálogo essencial de toda a equipe, além de incluir o paciente na terapêutica, pois ele sentirá que suas preocupações pessoais foram recebidas. É relevante o estudo sobre como realizar uma boa escuta, pois o processo de informação é um fator importante na assistência que irá ser prestada na individualidade de cada paciente ${ }^{(25)}$.

Entende-se a importância da escuta quando a comunicação com o paciente oncológico é colocado como uma estratégia fundamental para $\circ$ trabalho desenvolvido pela equipe de enfermagem. Os enfermeiros devem estar preparados para lidar com seus pacientes. Realizar uma escuta humanizada é também incluir o escutado no plano de cuidados. É tratá-lo como um sujeito responsável pela sua própria saúde, motivando-o assim, para o autocuidado. Valorizar a escuta, é uma intensificação das lutas diárias dessa nobre profissão(25).

\section{- Fatores capazes de dificultar e de qualificar a escuta no cuidado de enfermagem}

A má interação entre equipe-paciente ou equipe-família é um fator capaz dificultar o trabalho da enfermagem $^{(26)}$. A falta de tempo é uma reclamação constante dos profissionais, e é um obstáculo muito grande para a escuta. Infelizmente, esses não são os únicos fatores que desvalorizam esta ferramenta tão importante. $O$ número descabido e o preparo não adequado do pessoal causam a sobrecarga de quem deveria desempenhar com qualidade a escuta. $O$ nível de assistência é negativamente atingido nesses casos, pois a eficácia dos cuidados prestados aos pacientes oncológicos foram desqualificados. A identificação de possíveis outros problemas foram ignorados por alguma dessas razões, e futuramente podem resultar em um problema maior que poderia ter sido evitado pela escuta qualificada ${ }^{(27)}$.

Devido a uma rotina mecânica, a escuta frequentemente não é realizada como deveria. Por isso o relacionamento terapêutico vai depender diretamente das atitudes de interesse e do comportamento que o profissional decide ter. Contribui significativamente para a melhoria da assistência de enfermagem, considerar que a escuta é tão importante para a recuperação da saúde como também um tratamento medicamentoso. $O$ acolhimento e escuta devem ser associados como um cuidado de enfermagem, pois eles aceleram o processo de recuperação. Ou seja, minimizam o tempo de tratamento e ao mesmo tempo, maximizam a eficiência. Em contrapartida, o desconforto e a falta de confiança dos pacientes em compartilharem seus sentimentos podem ser fatores que não reduzem variáveis da ansiedade $\mathrm{e}$ do medo, ou seja, a escuta pode até ocorrer de forma adequada pelo profissional, porém depende de outros fatores relacionados à vontade do paciente ${ }^{(28)}$.

Estar aberto e apto para a escuta, separar tempo adequado, preparar o local para que tenha privacidade e não haja interrupções são cuidados fundamentais do enfermeiro com o seu paciente. $A$ habilidade de comunicação é utilizada como instrumento básico do enfermeiro para atender as necessidades de seus clientes com neoplasia. Tomar medidas para aprofundar o ato do cuidar é beneficiar aquele que se encontra a procura de informação e ajuda, pois teve sua qualidade de vida afetada pela enfermidade ${ }^{(29)}$. No ato do ouvir, o profissional terá sua primeira impressão, que incialmente será subjetiva, é a forma como ele enxerga seu cliente. Posteriormente poderão ser confirmadas por exame físico e exames clínicos. Por esta razão, ele deve valorizar as necessidades básicas apresentadas. A descrição das queixas devem ser avaliadas com atenção ${ }^{(30)}$.

Colocar o paciente oncológico como um ser importante para o profissional que o escuta, melhora a eficácia do diálogo, aumentando a coleta dos dados. À partir disso é possível implementar o plano de cuidado adequado, e quando colocado em prática: aliviará suas tensões fisiológicas e/ou emocionais. Isso acaba promovendo que o escutado aprenda a escutar outras pessoas também. Um profissional que não tem interesse no que o outro diz, infelizmente, não ouvirá alguém que precise de sua ajuda. Logo, esse profissional não está preparado para lidar com pessoas, embora tenha conhecimento científico, isso tudo será anulado quando não consegue interagir e se colocar no lugar de outros sujeitos. Estar ciente da importância que a escuta tem para si e para o outro, é o primeiro passo para se dispor a praticar a escuta ${ }^{(31)}$.

\section{CONCLUSÃO}

A literatura evidenciou que a escuta qualificada deve se fazer presente na rotina de todos os enfermeiros que atuam com os pacientes oncológicos, tendo em vista a quantidade de angústias e incertezas vivenciadas ao longo do tratamento, desde os primeiros sintomas e 0 momento do diagnóstico. Dessa forma, faz-se necessário valorizar e estimular a atuação do enfermeiro em praticar a escuta de forma adequada para atenuar essa problemática, que consequentemente, prejudica o estado clínico, a aceitação e a recuperação do paciente. Quando os pacientes oncológicos não conseguem adaptar-se à nova condição, surgem agravos psicológicos, que podem 
ser tão profundos, a ponto de afetarem a relação profissional-paciente, o que dificulta este à aderir as terapias e os tratamentos necessários à sua recuperação ou à sua melhoria da qualidade de vida.

Desse modo, divulgar os benefícios de uma escuta de qualidade se torna imprescindível na atuação do enfermeiro, pois o profissional necessita familiarizar-se com esse importante mecanismo e fundamentar suas ações em uma postura de quem ouve e entende o que se passa. Os estudos também evidenciaram que há obstáculos em intervir no manejo das angústias vivenciadas por esses pacientes, seja pela rotina mecânica ou sobrecarregada, cabendo especial papel ao profissional da Enfermagem como gerenciador e redutor dos sentimentos incertos.

Este trabalho proporciona, ao enfermeiro, o conhecimento de saber que a escuta é de sua área de atuação, bem como a análise detalhada de todos às informações advindas de uma fonte verbal ou não verbal relacionada ao paciente, e ao processo saúde-doença. É absolutamente essencial que, na busca de introduzir a escuta qualificada no cuidado clínico enfermagem ao paciente oncológico, estudos sejam realizados com o intuito de estimar a capacidade de atingir e a eficácia com qual atinge as boas práticas voltadas para essa implementação. 


\section{REFERÊNCIAS}

I Bray F, Ferlay J, Soerjomataram I, Siegel RL, Torre LA, Jemal A. Global cancer statistics 2018: GLOBOCAN estimates of incidence and mortality worldwide for 36 cancers in 185 countries. CA: a cancer journal for clinicians, Hoboken, v. 68, n. 6, p. 394-424, Nov. 2018.

2 Ribeiro JP, Cardoso LS, Pereira CMS, Silva BT, Bubolz BK, Castro CK. Assistência de enfermagem ao paciente oncológico hospitalizado: diagnósticos e intervenções relacionadas às necessidades psicossociais e psicoespirituais. Revista de Pesquisa [Internet]. 2016 [acesso em 16 nov 2020]; 8(4):5136-42. Disponível em: http://seer.unirio.br/index. php/cuidadofundamental/article/view/4016/pdf

3 Meneguin S, Matos TDS, Ferreira MLSM. Perception of cancer patients in palliative care about quality of life. Rev Bras Enferm [Internet]. 2018 [acesso em 25 out 2020]; 7I(4):1998-2004. Disponível em: http://www.scielo.br/pdf/ tce/v25nI/en_0104-0707-tce-25-0 I-33600 I4.pdf

4 Markus LA, Betiolli SE, Souza SJP, Marques FR, Migoto MT. $A$ atuação do enfermeiro na assistência ao paciente em cuidados paliativos. Rev. Gestão E Saúde [Internet]. 2017; [acesso em 25 out 2020]; I7(Supl.I):7I-8I. Disponível em: http://www.herrero.com.br/files/revista/file808a997f5fc0c522425922dc99ca39b7.pdf

5 Menezes EMPP, Lagemann RAP, Lagemann VD, Lagemann RC, Bruno KRG.A relação enfermeiro-paciente oncológico em uma abordagem holística. Rev Cient Fac Educ e Meio Ambient [Internet]. 2020 [acesso em 16 nov 2020]; I0(Especial): I I-5. Disponível em: http://www.faema.edu.br/revistas/index.php/Revista-FAEMA/article/view/90I

6 Assis MMA, Nascimento MAA, Pereira MJB, Cerqueira EM. Cuidado integral em saúde: dilemas e desafios da enfermagem. Rev Bras Enferm[Internet]. 2015[acesso em 16 nov 2020];68(2):333-8. Disponível em: http://www.scielo.br/ pdf/reben/v68n2/0034-7167-reben-68-02-0333.pdf

7 Instituto Nacional de Câncer [homepage na internet]. Estatísticas de Câncer: Incidência estimada conforme a localização primária do tumor e sexo [acesso em 04 set 2020]. Disponível em: https://www.inca.gov.br/numeros-de-cancer

8 Leite MA, Nogueira DA, Terra F de S. Evaluation of self-esteem in cancer patients undergoing chemotherapy treatment. Rev Lat Am Enfermagem [Internet]. 2015 [acesso em 25 out 2020]; 23(6):1082-1089. Disponível em: http://www.scielo.br/scielo.php?script=sci_arttext\&pi$d=S 0104-1$ I 6920 I 500060 I 082\&lng=en.

9 Teston EF, Fukumori EFC, Benedetti GMS, Spigolon DN, Costa MAR, Marcon SS. Feelings and difficulties experienced by cancer patients along the diagnostic and therapeutic itineraries. Esc Anna Nery [Internet]. 2018 [acesso em 25 out 2020]; 22(4):e20180017. Disponível em: http://www.scielo.br/scielo.php?script=sci_arttext\&pi$\mathrm{d}=\mathrm{S}|4| 4-8|4520| 80004002 \mid 4 \&$ Ing=en.

10 Mendes KDS, Silveira RCCP, Galvão CM. Revisão integra- tiva: método de pesquisa para a incorporação de evidências na saúde e na enfermagem. Texto contexto - enferm. [Internet]. 2008 [acesso em 21 abr 2020]; 17(4):758-764. Disponível em: https://www.scielo.br/pdf/tce/v17n4//8.pdf.

II Oxford Centre for Evidence-based Medicine: levels of evidence [Internet]. 2009 [acesso em 25 out 2020]. Disponível em: http://www.cebm.net/oxford-centre-evidence-basedmedicine-levels-evidence-march-2009

12 Pereira MG, Galvão TF. Etapas de busca e seleção de artigos em revisões sistemáticas da literatura. Epidemiol. Serv. Saúde [Internet]. 2014 [acesso em 25 out 2020]; 23(2):36937I. Disponível em: http://scielo.iec.gov.br/scielo.php?scrip$\mathrm{t}=\mathrm{sci}$ _arttext\&pid=SI 679-497420 I 40002000 I 9\&Ing=pt.

13 Moher D, Liberati A, Tetzlaff J, Altman DG; PRISMA Group. Preferred reporting items for systematic reviews and meta-analyses: the PRISMA statement. PLoS Med [Internet]. 2009 [acesso em 06 jul 2020]; 6(7):el000097. Disponível em: https://journals.plos.org/plosmedicine/article?id=10.137| /journal.pmed. 1000097

I4 Galvão TF, Pereira MG. Avaliação da qualidade da evidência de revisões sistemáticas. Epidemiol. Serv. Saúde [Internet]. 2015 [acesso em 25 out 2020]; 24(I):I73-I75. Disponível em: http://scielo.iec.gov.br/scielo.php?script=sci_arttext\&pid=S1679-497420 I5000 I000 I 9\&Ing=pt.

I5 Beltrán-Salazar O.Atención al detalle, un requisito para el cuidado humanizado. Index Enferm [Internet]. 2015 [acesso en 28 set 2020]; 24(I-2):49-53. Disponível em: http://scielo.isciii.es/scielo.php?script=sci_arttext\&pi$d=S \mid$ I 32- I 29620 I 5000 I 000 I I \&Ing=es.

16 Garcia ACM, Simão-Miranda TP, Carvalho AMP, Elias PCL, Pereira MG, Carvalho EC. The effect of therapeutic listening on anxiety and fear among surgical patients: randomized controlled trial. Rev. Latino-Am. Enfermagem [Internet]. 2018 [acesso em 28 set 2020]; 26: e3027. Available from: http://www.scielo.br/scielo.php?script=sci_arttext\&pid=SO I 04- I I 6920 I $800010034 \mid$ \&lng=en

17 Ferreira $\mathrm{ABH}$. Novo dicionário da língua portuguesa. 2a.ed. Rio de Janeiro: Nova Fronteira; 1986.

I8 Anacleto G, Cecchetto FH, Riegel F. Cuidado de enfermagem humanizado ao paciente oncológico: revisão integrativa. Rev Enferm Contemp [Internet]. 2020 [acesso em 28 set 2020]; 9(2):246-254. Disponível em: https://www5.bahiana. edu.br/index.php/enfermagem/article/view/2737/3 II4

19 Wakiuchi J, Oliveira DC, Marcon SS, Oliveira MLF, Sales CA. Meanings and dimensions of cancer by sick people - a structural analysis of social representations. Rev. esc. enferm. USP [Internet]. 2020 [acesso em 25 out 2020]; 54:e03504. Disponível em: http://www.scielo.br/scielo.php?script=sci_arttext\&pid=S0080-62342020000 $100408 \&$ Ing=en.

20 Catapreta AA, Denadai W, Marcial VMV, Matos FS, Coelho SC, Ardisson MD, Faria RA. A comunicação na unidade de terapia intensiva oncológica: Uma revisão sistemática sobre os vieses que interferem e ou participam na comunicação 
entre enfermeiros e pacientes oncológicos. Brazilian Journal of Health Review [Internet]. 2020 [acesso em 25 out 2020]; 3(4): I0487-I0500. Disponível em: https://www.brazilianjournals.com/index.php/BJHR/article/view/I5059

2I Nascimento CCN. Influências sociais e emocionais da humanização a assistência ao paciente oncológico. Revista Contribuciones a las Ciencias Sociales [Internet]. 2019 [acesso em 25 out 2020]. Disponível em: https://www.eumed.net/rev/cccss/2019/07/assistencia-paciente-oncologico.html//hdl.handle.net

22 Theobald MR, Santos MLM, Andrade SMO, Carli AD. Percepções do paciente oncológico sobre o cuidado. Physis [Internet]. 2016 [acesso em 25 out 2020]; 26(4): I 249- 1269. Disponível em: http://www.scielo.br/scielo.php?script=sci_ arttext\&pid=SO $03-733|201600040| 249 \&$ Ing=en.

23 Vargas $\mathrm{Cl}$, Concha MC. Moral Distress, Sign of Ethical Issues in the Practice of Oncology Nursing: Literature Review.Aquichan [Internet]. 2019 [acesso em 25 out 2020]; I9(I):II-I5. Disponível em: http://www.scielo.org.co/scielo.php?script=sci_arttext\&pid=SI 657-599720 I 90001000 I I \&lng=en.

24 Lira BSM,Andrade EGS.Assistência do enfermeiro no atendimento domiciliar em pacientes oncológicos. Rev Inic Cient e Ext [Internet]. 2019 [acesso em 25 out 2020];2(Esp.2):3 I4-22. Disponível em: https://revistasfacesa.senaaires.com.br/index. php/iniciacao-cientifica/article/view/28I

25 Fontes CMB, Menezes DV, Borgato MH, Luiz MR. Communicating bad news: an integrative review of the nursing literature. Rev Bras Enferm [Internet]. 2017 [acesso em 25 out 2020]; 70(5):1089-1095. Disponível em: http://www.scielo.br/scielo.php?script=sci_arttext\&pi$d=$ S0034-7 I 6720 I 700050 I 089\&Ing=en

26 Brandão MLA, Góis RMO. Assistência de enfermagem para pacientes oncológicos em cuidados paliativos: importância da interação familiar no tratamento. Caderno de Graduação-Ciências Biológicas e da Saúde-UNIT-SERGIPE [Internet]. 2020 [acesso em 25 out 2020]; 6(I): 175. Disponível em: https://periodicos.set.edu.br/cadernobiologicas/article/view/8180

27 Sant'ana JLG, Maldonado MU, Gontijo LA. Dinâmica de geração e dissipação do estresse na equipe de enfermagem num centro de oncologia. Rev. Latino-Am. Enfermagem [Internet]. 2019 [acesso em 25 out 2020]; 27:e3156. Disponível em: http://www.scielo.br/scielo.php?script=sci_arttext\&pi$\mathrm{d}=$ S0 I 04- I I 6920 I 9000 I00342\&Ing=en. Epub July 18, 2019.

28 Domenico EBL. A complexidade do cuidado em oncologia: desafios atuais e futuros. Acta Paul Enferm [Internet]. 2016 [acesso em 25 out 2020]; 29(3):3-5. Disponível em: http://www.scielo.br/scielo.php?script=sci_arttext\&pi$d=S 0103-21002016000300003 \& \mid n g=e n$.

29 Picollo DP, Fachini M.A atenção do enfermeiro ao paciente em cuidado paliativo. Revista de Ciências Médicas [Internet]. 2018 [acesso em 25 out 2020]; 27(2):85-92. Disponível em: http://dx.doi.org/ I0.24220/23 I8- 0897v27n2a3855

30 Ugarte GMI. Responsabilidad en los cuidados enfermeros: poniendo en el centro a la persona. Rev Enf Ref [Internet]. 2018 [acesso em 25 out 2020]; serIV(I7): I6I-169. Disponível em: http://www.scielo.mec.pt/scielo.php?script=sci_arttext\&pid=S0874-028320 I $8000200016 \&$ Ing=pt.

3I Luz KR, Vargas MAO, Barlem ELD, Schmitt PH, Ramos FRS, Meirelles BHS. Estratégias de enfrentamento por enfermeiros da oncologia na alta complexidade. Rev Bras Enferm [Internet]. 2016 [acesso em 25 out 2020]; 69(I):67-7I. Disponível em: http://www.scielo.br/scielo.php?script=sci_arttext\&pid=S0034-7| $672016000100067 \&$ Ing=en.

Recebido: 2020-10-30

Aceito: $2020-12-07$ 